\title{
Surface antiferromagnetism and incipient metal-insulator transition in strained manganite films
}

\author{
F. Cossu, ${ }^{1}$ U. Schwingenschlögl, ${ }^{1}$ G. Colizzi, ${ }^{2}$ A. Filippetti, ${ }^{2}$ and Vincenzo Fiorentini ${ }^{2}$ \\ ${ }^{1}$ Materials Science and Engineering, KAUST, 23955-6900 Thuwal, Kingdom of Saudi Arabia \\ ${ }^{2}$ CNR-IOM and Dipartimento di Fisica, Università di Cagliari, I-09042 Monserrato (Ca), Italy \\ (Received 20 December 2012; revised manuscript received 7 May 2013; published 21 June 2013)
}

\begin{abstract}
Using first-principles calculations, we show that the (001) surface of the ferromagnet $\mathrm{La}_{0.7} \mathrm{Sr}_{0.3} \mathrm{MnO}_{3}$ under an epitaxial compressive strain favors antiferromagnetic (AF) order in the surface layers, coexisting with ferromagnetic (FM) bulk order. Surface antiferromagnetism is accompanied by a very marked surface-related spectral pseudogap, signaling an incomplete metal-insulator transition at the surface. The different relaxation and rumpling of the $\mathrm{MnO}_{2}$ and $\mathrm{LaO}$ surface planes in the two competing magnetic phases cause distinct work-function changes, which are of potential diagnostic use. The AF phase is recognized as an extreme surface-assisted case of the combination of in-plane AF super-exchange and vertical FM double-exchange couplings that rules magnetism in manganites under in-plane compression.
\end{abstract}

DOI: 10.1103/PhysRevB.87.214420

PACS number(s): 73.20.-r, 75.70.Ak, 75.47.Lx, 75.70.Rf

Rare-earth manganites are promising for applications to magnetic sensors, ${ }^{1,2}$ spintronic devices, ${ }^{3-8}$ and fuel-cell components, ${ }^{9,10}$ and have been the focus of many theoretical studies. ${ }^{11-14}$ The colossal-magnetoresistant aboveroom-temperature ferromagnet (FM) $\mathrm{La}_{1-x} \mathrm{Sr}_{x} \mathrm{MnO}_{3}$ (LSMO henceforth) around $x=1 / 3$ is one of the most studied in this class. Its magnetic phase and structural properties are quite sensitive to hydrostatic as well as uniaxial strains. ${ }^{13,14}$ It is natural to expect that such effects will be amplified at surfaces and that tight magnetic phase competition may ensue, as suggested by previous theoretical work ${ }^{15-17}$ and by experimental observations of a lowered magnetic moment in substrate-constrained LSMO films. ${ }^{18,19}$ Here we demonstrate, using $a b$ initio simulations, that indeed antiferromagnetism (AF) takes over at the (001) surface of LSMO under appropriate in-plane compression, and it coexists with FM order inside the bulk. The surface exhibits large rumpling relaxations which affect the work function, and its electronic properties suggest an incipient surface metal-insulator transition and reduced in-plane conductivity.

First-principles calculations are performed using the projector-augmented wave method ${ }^{20}$ within the generalized gradient approximation to density functional theory with Hubbard correction ${ }^{21}(\mathrm{GGA}+\mathrm{U})$ in the Dudarev formulation ${ }^{22}$ as implemented in the VASP code. ${ }^{23}$ The only independent parameter, U-J, is set to $2 \mathrm{eV}$ as in our previous work on bulk LSMO. ${ }^{13,14} \mathrm{GGA}+\mathrm{U}$ opens a gap at the Fermi level $\left(E_{F}\right)$ in the minority channel, ${ }^{13,14,24}$ in agreement with the known semimetallic character of LSMO. In the plain gradient approximation (GGA), $E_{F}$ barely slices through the lower minority conduction band, so the GGA structure and magnetism are in fact largely similar to GGA $+\mathrm{U}$, and are not discussed further here. The energy cutoff is $400 \mathrm{eV}$, and $k$-point grids up to $9 \times 9 \times 2$ are used.

We simulate the epitaxially constrained Mn-terminated (001) LSMO surface with a 13-layer symmetric slab with $\sqrt{2} \times \sqrt{2}$ (two cations per plane) in-plane section, and containing seven $\mathrm{MnO}_{2}$ and six $(\mathrm{La} / \mathrm{Sr}) \mathrm{O}$ layers at different inplane strains. Doping at $x=1 / 3$ is effected by $\mathrm{Sr}$ substitution of one $\mathrm{La}$ per $\mathrm{LaO}$ layer, symmetrically with respect to the central $\mathrm{MnO}_{2}$ layer; the subsurface $\mathrm{LaO}$ layers are $\mathrm{Sr}$-free.
The periodic slab images are separated by $\simeq 10 \AA$ of vacuum. All ionic degrees of freedom are relaxed starting from the bulk rhombohedral $R 3 c$ structure, with force threshold $0.01 \mathrm{eV} / \AA$.

With a fairly extensive search for competing surface magnetic structures, we restricted the comparison to the FM, $\mathbf{q}=(0,0,0)$, and to structures we call " $1 \mathrm{f}$ " and " $2 \mathrm{f}$ ", namely FM phases with $\mathrm{Mn}$ moments in the surface and, respectively, subsurface $\mathrm{MnO}_{2}$ layer being spin-flipped according to an $\mathrm{AF}_{C}$ pattern with $\mathbf{q}=(1 / 2,1 / 2,0)$ as in Fig. 1 , rightmost panel. Magnetic patterns which are not energy competitive are the same as $1 \mathrm{f}$ or $2 \mathrm{f}$ with three or more Mn layers flipped, and others locally mimicking bulk $\mathrm{AF}_{A}[\mathbf{q}=(0,0,1 / 2)]$ and $\mathrm{AF}_{G}[\mathbf{q}=(1 / 2,1 / 2,1 / 2)]$. The magnetic behavior is found to be robust with respect to slab thickness (from 7 layers to the 13 layers used here).

The energies of $1 \mathrm{f}$ and FM vs strain are shown in Fig. 2. Zero strain corresponds to the experimental bulk lattice constant of LSMO $(3.871 \AA)$. The results indicate a stability region for If at strains around $-2.5 \%$. At larger compressive strains the FM phase becomes favored again (although more complex AF phases ${ }^{18}$ not considered here may start playing a role), whereas for tensile strains no competition occurs: the FM is always favored. Figure 2 also shows that in both phases the surface at zero strain is subject to a tensile surface stress. A rough estimate from the linear term of the energy $\mathrm{y}^{25}$ around zero strain gives a surface stress of $0.20 \mathrm{eV} / \AA^{2}$, which is comparable to the very large $e^{26} 0.35 \mathrm{eV} / \AA^{2}$ of Pt (001). The finite-thickness simulation slab is contracted by the surface stress, and is in equilibrium at a nonzero compressive strain, which indeed corresponds to the release of a tensile stress. Thus, LSMO thin films will adapt more easily to shorter-lattice-constant substrates, less so to larger-lattice-constant ones. (Specifically, at the present film thickness of about $1 \mathrm{~nm}$ the effective strain needed to stabilize the $1 \mathrm{f}$ structure is about $-1.5 \%$.)

The raw total energies of $1 \mathrm{f}$ and FM (Fig. 2) show that in the vicinity of $-2.5 \%$ strain the first surface layer of LSMO (001) will be antiferromagnetic. Below we analyze the structure and magnetic properties at this strain, which, we note, corresponds to growth on $\mathrm{LaAlO}_{3}$. While the 1f (top-layer $\mathrm{AF}$ ) phase is favored, the $2 \mathrm{f}$ phase is disfavored, indicating that $\mathrm{AF}$ will hardly penetrate deeper than one Mn layer into the bulk. Even 


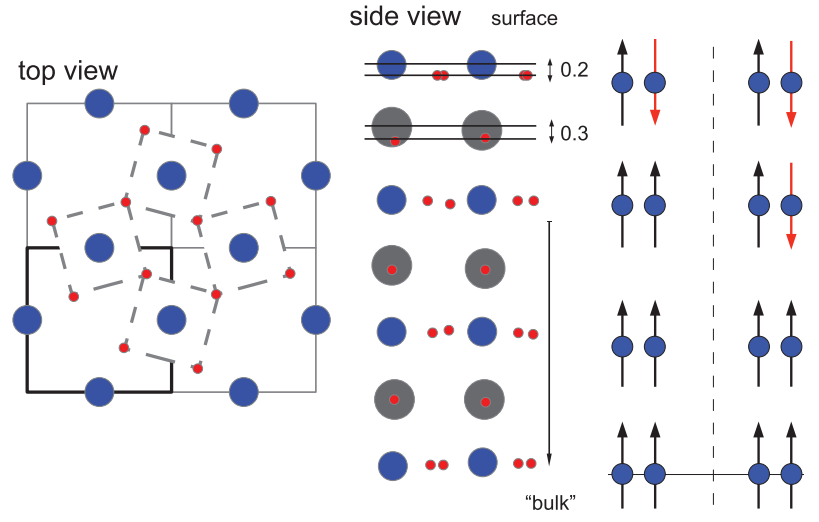

FIG. 1. (Color online) Sketch of the simulation supercell (small red circles: O; large gray circles: $\mathrm{La} / \mathrm{Sr}$; intermediate blue circles: $\mathrm{Mn})$. Left: top view (in-plane surface cell outlined). Center: side view (including vertical rumpling of surface layers). Right: $1 \mathrm{f}$ and $2 \mathrm{f}$ magnetic structures (surface is upper side).

if we factor out the bulk energy using a surface-specific energy gain $\Delta \gamma=\left(\Delta E_{s}-n \Delta E_{b}\right) / 2 A$ in lieu of the raw energy difference $\left(\Delta E_{s}\right.$ is the energy difference between the FM slab and the spin-flipped slab, $\Delta E_{b}$ is twice-there being two Mn per layer-the bulk energy difference per formula unit between $\mathrm{AF}_{C}$ and FM, $n$ the number of spin-flipped layers, $A=28.6 \AA^{2}$ the surface area), the $2 \mathrm{f}$ structure still gains zero energy to numerical accuracy, and all other structures give negative gains. This confirms that only the topmost layer of the surface region of LSMO (001) is likely to exhibit antiferromagnetism. Indeed, AF is expected to be surface induced and surface confined: a previous analysis ${ }^{27}$ of bulk LSMO under strain has shown that the vertical magnetic couplings are large and positive (i.e., FM) for all reasonable epitaxial conditions and external strains. A corollary, confirmed by the calculation, is that larger strains will not help AF to dive any deeper into the bulk.

The strained surface exhibits quite outstanding features for a metallic surface: (i) a very large outward relaxation of the

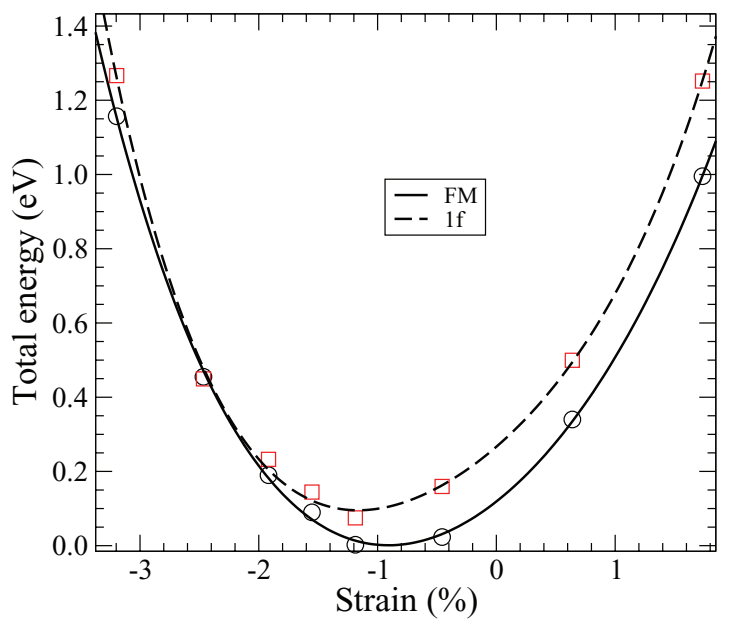

FIG. 2. (Color online) Calculated energies vs strain for the FM (circles) and 1f (squares) structures. Lines are fourth-order polynomial fits; the energy zero is the minimum of the FM fit.

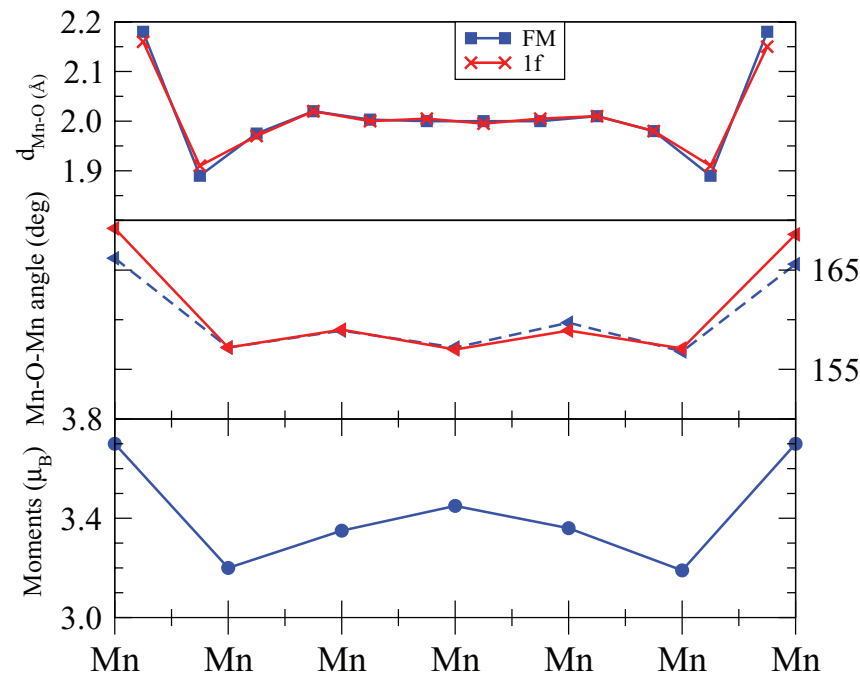

FIG. 3. (Color online) Structural parameters vs position (top surface: left; bottom surface: right). Top: Mn-apical O interlayer distance; center: octahedron rotation arouns $\hat{s}$; bottom, Mn magnetic moments.

top layer (almost 20\% compared to bulk interlayer spacing), associated with (ii) an increased $(+10 \%) \mathrm{Mn}$ magnetic moment at the surface, and (iii) a large rumpling (over $10 \%$ on average) in the top $\mathrm{MnO}_{2}$ and $\mathrm{LaO}$ layers. A selection of calculated quantities is in Fig. 3. Mn-O octahedra behave nearly as rigid units, except in the surface and subsurface layers, where structural and magnetic behavior change in lockstep. The tilt angles of the octahedra off the surface normal $\hat{s} \equiv(001)$ are essentially zero all over the slab, i.e., octahedra are perfectly aligned along $\hat{s}$ and laterally rigid. The large rotations of the octahedra around $\hat{s}$ are roughly constant in the bulk region, with a Mn-O-Mn angle $\alpha=160^{\circ}$ as in bulk LSMO at the same strain; the rotation then increases in the subsurface layer $\left(\alpha=155^{\circ}\right)$, and finally decreases in the surface layer $\left(\alpha=170^{\circ}\right)$. Concurrently, the vertical (i.e., along $\hat{s}$ ) distance between $\mathrm{Mn}$ in the top layer and $\mathrm{O}$ in the $\mathrm{LaO}$ layer just below increases to $2.2 \AA(+10 \%$ relative to the bulk $2.0 \AA)$, while that between the subsurface $\mathrm{Mn}$ and $\mathrm{O}$ in the $\mathrm{LaO}$ layer below drops to $1.9 \AA(-5 \%)$. This correlates with the Mn magnetic moment, which drops from $3.4 \mu_{B}$ in the bulk to $3.3 \mu_{B}$ in the subsurface layer $(-6 \%)$, and then increases to $3.7 \mu_{B}$ in the top layer $(+9 \%)$.

In addition, the $\mathrm{MnO}_{2}$ surface plane is rumpled, as sketched in Fig. 1 and summarized in Table I, with Mn atoms being strongly displaced upwards relative to $\mathrm{O}$ atoms. The same pattern, even in quantitative terms, appears in the first $\mathrm{LaO}$ layer from the surface. The rumpling is very large: the $\mathrm{Mn}-\mathrm{O}$ (La-O) height difference is over two (over twenty) times the analogous $\mathrm{Ti}-\mathrm{O}(\mathrm{Pb}-\mathrm{O})$ displacement in the typical ferroelectric ${ }^{28}$ titanate $\mathrm{PbTiO}_{3}$. This displacement generates a

TABLE I. Rumpling $(\AA)$ of the top surface layers.

\begin{tabular}{lccc}
\hline \hline & FM & AF & $\delta$ \\
\hline $\mathrm{MnO}_{2}$ & 0.235 & 0.170 & -0.065 \\
$\mathrm{LaO}$ & 0.265 & 0.255 & -0.010 \\
\hline \hline
\end{tabular}




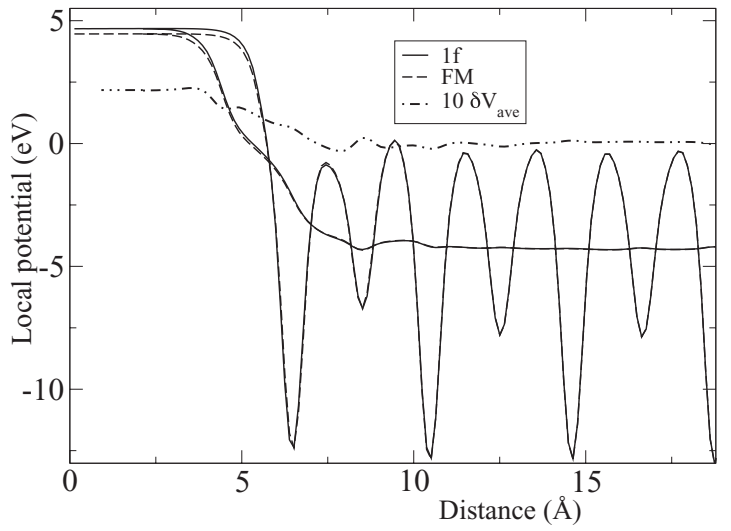

FIG. 4. Local potentials and their periodicity-filtering running averages for FM (dashed) and 1f surfaces (solid) between surface (left) and slab center (right). Energy zero is the Fermi energy. The dash-dot line at the center is the difference $\delta \mathrm{V}_{\text {ave }}$ of the $1 \mathrm{f}$ and $\mathrm{FM}$ averages (amplified by a factor 10).

large surface dipole pointing out of the solid into the vacuum. In particular, the FM surface has a larger near-surface rumpling than the AF surface (Table I; effectively, the only significant structural difference between the two). This is mirrored in a reduced work function of the FM ( $W=4.47 \mathrm{eV}$ for FM and $W=4.67 \mathrm{eV}$ for AF). Of course, this matches the usual trend of metal work function lowering with increased surface corrugation. Based on this result, a work function increase in a strained LSMO film would be a diagnostic evidence of a FM-to-AF magnetic phase change.

This argument is validated by Fig. 4, which displays the local potentials and their periodicity-filtering running averages for the FM (dashed) and 1f surfaces (solid). The local potential has deep troughs at the atomic positions (the deepest ones are $\mathrm{Mn}$, the others $\mathrm{La}$ ), while the filtered averages are about constant in the bulk and in the vacuum. The dash-dot line at the center is the difference $\delta \mathrm{V}_{\text {ave }}$ of the 1f and FM averages amplified by a factor 10 ; clearly $\delta \mathrm{V}_{\text {ave }}$ is zero in the bulk, it starts being perturbed by the surface at the second Mn layer, it picks up decidedly from zero just below the top Mn layer as expected from the dipole difference, it grows steadily as the surface is crossed, and finally saturates to the work function difference $(0.2 \mathrm{eV})$ in the vacuum.

The electronic structure signature of this "ferroelastic"17 distortion is a surface weakening of the directional $d_{z^{2}-}$ mediated bonding, as borne out by Fig. 5, discussed below. A similar rumpling of $\mathrm{MnO}_{2}$ planes (La-O layer rumplings were not mentioned) at FM, unstrained LSMO (001) was reported earlier ${ }^{17}$ to decay gradually into the bulk. In the present case of in-plane compression this feature is much stronger and more surface localized: our rumpling is twice as large $(0.27 \AA$ vs $0.14 \AA$ ), and it occurs only in the outermost $\mathrm{MnO}_{2}$ and $\mathrm{LaO}$ layers, while it is an order of magnitude lower, i.e., negligible, in the other layers.

Figure 5 reports the orbitally resolved density of states (DOS) projected on Mn for the FM (left) and 1f (right) magnetic order, at the surface (top) and in the innermost layer (bottom). The bulk DOS in the lower panels is identical in the two cases, demonstrating further that the AF surface does not affect bulk magnetism beyond at most $1 \mathrm{~nm}$ depth; also,
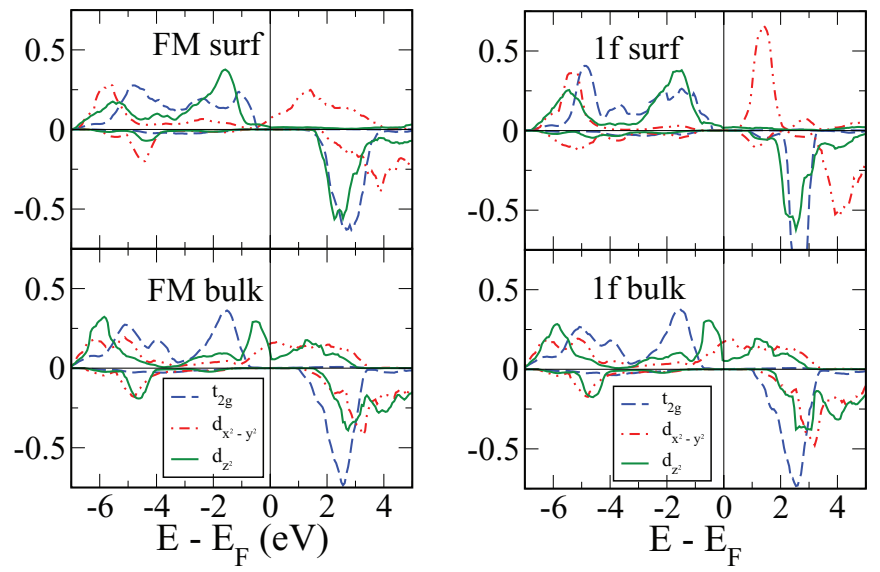

FIG. 5. (Color online) Orbitally resolved DOS for Mn at the surface (top) and in the innermost layer (bottom) in the FM (left) and If (right) magnetic order. Majority (minority) spin is positive (negative). Both surfaces are metallic, but the $1 \mathrm{f}$ has a much smaller DOS. The bulk DOS is identical in the two cases, demonstrating that the AF surface does not affect bulk magnetism beyond about $1 \mathrm{~nm}$ depth.

half-metallicity (a gap in the minority channel) is preserved in all cases both in the bulk and at the surface. While the bulk is metallic with roughly equal participation of both $e_{g}$ orbitals, the FM surface is still fully metallic, but with mostly $d_{x^{2}-y^{2}}$ and only residual $d_{z^{2}}$ character at $E_{F}$. The standard DOS narrowing and upward shift at the surface (which would provide strong $d_{z^{2}}$ metallicity) is prevented by the huge outward relaxation, which indeed is associated with the $d_{z^{2}}$ peak dipping below $E_{F}$. The AF $1 f$ surface has a real gap in both the $t_{2 g}$ and $d_{x^{2}-y^{2}}$ channels. On the other hand, the $d_{z^{2}}$ weight moves below $E_{F}$ as in the FM, but this demotion is incomplete and a small but appreciable DOS survives at $\mathrm{E}_{F}$. We thus end up with a deep surface-related pseudogap, signaling an incipient but incomplete metal-insulator transition at the surface. This is still technologically relevant, since conduction through a contact on this surface in, e.g., a tunneling-magnetoresistance or field-effect device would clearly be affected by this 1-eV-wide pseudogap.

The marginal (in the sense of being related to a pseudogap, for example as in bulk Be) character of surface metallicity in the surface AF phase is further illuminated by the orbitally resolved DOS for the spin-up $\mathrm{Mn}$ in the AF top layer and the corresponding apical oxygen $\mathrm{O}_{a}$ in the subsurface $\mathrm{LaO}$ layer, displayed in Fig. 6, bottom. Clearly, the residual metallic character is due to the residual (nonbonding) overlap of $\mathrm{Mn}$ $d_{z^{2}}$ with $\mathrm{O}_{a} p_{z}$ in the majority channel (solid lines), which confirms that the in-plane orbital channels are all gapped. The spin-down $\mathrm{Mn}$ and corresponding $\mathrm{O}_{a}$ (not shown) behave similarly, with a nonzero DOS in the majority channel, that preserves the (by now weak) semimetallic character. Despite the absence of a proper gap, it is likely that the conductivity will be strongly diminished, especially in-plane, due to the drop in the DOS at $E_{F}$.

In closing, we note that the surface electronic structure just discussed is fully consistent with the strain effects on magnetic coupling mechanisms discussed in Ref. 14 (see in particular the left panel of Fig. 14 thereof): basically, in-plane 


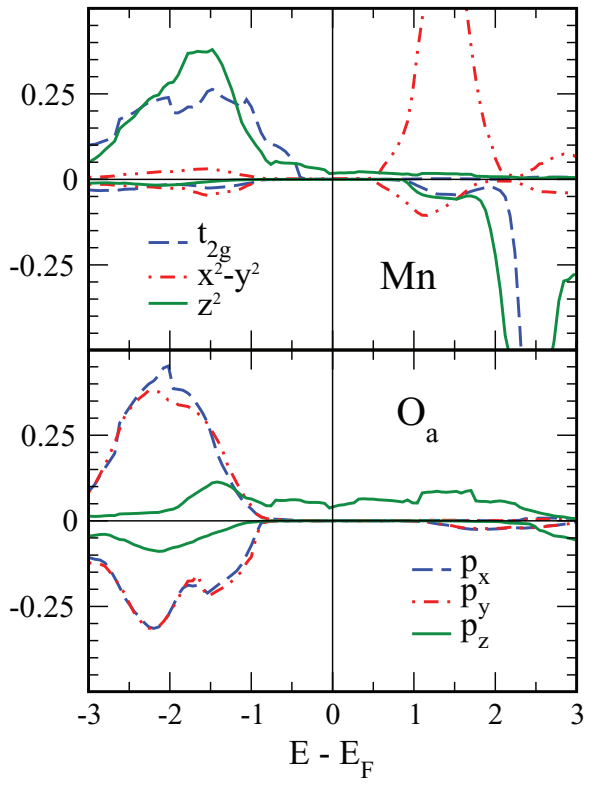

FIG. 6. (Color online) A closeup view in the Fermi level region of the DOS projected on the spin-up surface Mn and the corresponding apical subsurface $\mathrm{O}$ for the $1 \mathrm{f}$ structure (see also Fig. 5, top right). The residual metallic character is due to the vertical, nonbonding overlap of Mn $d_{z^{2}}$ with $\mathrm{O}_{a} p_{z}$.

compression favors rotations around the vertical axis $\hat{s}$, and unbalances the magnetic couplings so that double-exchange
(FM) tends to prevail along $\hat{s}$ while super-exchange (AF) is stronger in-plane (also, a charge transfer from $d_{x^{2}-y^{2}}$ to $d_{z^{2}}$ attends to this shift). However, very large strains would be needed in the bulk for AF to prevail; the surface provides locally the additional structural freedom to (barely) tip the balance in AF's favor in some strain range, realizing locally a forerunner of magnetic phase separation. ${ }^{18}$

In summary, we have shown that under compressive epitaxial strain the first Mn surface layer (possibly, at most, the first two) of ferromagnetic LSMO (001) turns antiferromagnetic. This change in magnetic order is accompanied by a marked surface-related spectral pseudogap, signaling an incomplete metal-insulator transition at the surface. The LSMO surface is under significant tensile stress, which favors accommodation on compressing epitaxial substrates. The $\mathrm{MnO}_{2}$ and $\mathrm{LaO}$ surface planes are severely rumpled due to weakened $\mathrm{Mn} d_{z^{2}-\mathrm{O}}$ $p_{z}$ bonding, and the different rumpling in the two magnetic phases causes a distinct work function shift of potential diagnostic use. The surface AF phase is an extreme surfaceassisted case of a previously recognized ${ }^{14}$ combination of $^{4}$ in-plane AF super-exchange and vertical FM double-exchange couplings under in-plane compression.

This work was supported in part by projects EU FP7 OxIDes (Grant No. 228989), MIUR-PRIN 2008 2-DEG FOXI and 2010 Oxide, IIT Seed NEWDFESCM, Fondazione Banco di Sardegna 2011. Computational resources were provided by CASPUR Rome and by KAUST HPC.
${ }^{1}$ S. Jin, M. McCormack, T. H. Tiefel, and R. Ramesh, J. Appl. Phys. 76, 6929 (1994).

${ }^{2}$ L. I. Balcells, R. Enrich, J. Mora, A. Calleja, J. Fontcuberta, and X. Obradors, Appl. Phys. Lett. 69, 1486 (1996).

${ }^{3}$ I. Pallecchi, L. Pellegrino, E. Bellingeri, A. S. Siri, and D. Marré, Phys. Rev. B 71, 014406 (2005); J. Appl. Phys. 95, 8079 (2004); Appl. Phys. Lett. 83, 4435 (2003).

${ }^{4}$ L. E. Hueso, J. M. Pruneda, V. Ferrari, G. Burnell, J. P. Valdés-Herrera, B. D. Simons, P. B. Littlewood, E. Artacho, A. Fert, and N. D. Mathur, Nature (London) 445, 410 (2007).

${ }^{5}$ H. J. Liu, S. L. Lim, and C. K. Ong, Mater. Lett. 62, 2006 (2008).

${ }^{6} \mathrm{~S}$. Mathews, R. Ramesh, T. Venkatesen, and J. Benedetto, Science 276, 238 (1997).

${ }^{7}$ A. J. Millis, Nature (London) 392, 147 (1998).

${ }^{8}$ H. Q. Yin, J. S. Zhou, and J. B. Goodenough, Appl. Phys. Lett. 77, 714 (2000).

${ }^{9}$ A. Lussier, J. Dvorak, S. Stadler, J. Holroyd, M. Liberati, E. Arenholz, S. B. Ogale, T. Wu, T. Venkatesan, and Y. U. Idzerda, Thin Solid Films 516, 880 (2008).

${ }^{10}$ K. Miyazaki, N. Sugimura, K. Matsuoka, Y. Iriyama, T. Abe, M. Matsuoka, and Z. Ogumi, J. Power Sources 178, 683 (2008).

${ }^{11}$ Y. Tokura and N. Nagaosa, Science 288, 462 (2000).

${ }^{12} \mathrm{~S}$. Yunoki, J. Hu, A. L. Malvezzi, A. Moreo, N. Furukawa, and E. Dagotto, Phys. Rev. Lett. 80, 845 (1998).

${ }^{13}$ G. Colizzi, A. Filippetti, and V. Fiorentini, Phys. Rev. B 76, 064428 (2007).

${ }^{14}$ G. Colizzi, A. Filippetti, F. Cossu, and V. Fiorentini, Phys. Rev. B 78, 235122 (2008).
${ }^{15}$ A. Filippetti and W. E. Pickett, Phys. Rev. Lett. 83, 4184 (1999).

${ }^{16}$ A. Filippetti and W. E. Pickett, Phys. Rev. B 62, 11571 (2000).

${ }^{17}$ J. M. Pruneda, V. Ferrari, R. Rurali, P. B. Littlewood, N. A. Spaldin, and E. Artacho, Phys. Rev. Lett. 99, 226101 (2007).

${ }^{18}$ A. Tebano, C. Aruta, P. G. Medaglia, F. Tozzi, G. Balestrino, A. A. Sidorenko, G. Allodi, R. De Renzi, G. Ghiringhelli, C. Dallera, L. Braicovich, and N. B. Brookes, Phys. Rev. B 74, 245116 (2006).

${ }^{19}$ A. Geddo Lehmann, C. Sanna, N. Lampis, F. Congiu, G. Concas, L. Maritato, C. Aruta, and A. Yu. Petrov, Eur. Phys. J. B 55, 337 (2007).

${ }^{20}$ P. E. Blöchl, Phys. Rev. B 50, 17953 (1994); G. Kresse and D. Joubert, ibid. 59, 1758 (1999).

${ }^{21}$ V. I. Anisimov, J. Zaanen, and O. K. Andersen, Phys. Rev. B 44, 943 (1991).

${ }^{22}$ S. L. Dudarev, G. A. Botton, S. Y. Savrasov, C. J. Humphreys, and A. P. Sutton, Phys. Rev. B 57, 1505 (1998).

${ }^{23}$ G. Kresse and J. Furthmüller, Phys. Rev. B 54, 11169 (1996).

${ }^{24}$ C. Ma, Z. Yang, and S. Picozzi, J. Phys.: Condens. Matter 18, 7717 (2006).

${ }^{25}$ P. M. Marcus, X. Qian, and W. Hübner, J. Phys.: Condens. Matter 12, 5541 (2000).

${ }^{26}$ V. Fiorentini, M. Methfessel, and M. Scheffler, Phys. Rev. Lett. 71, 1051 (1993).

${ }^{27}$ G. Colizzi, A. Filippetti, F. Cossu, and V. Fiorentini, Eur. Phys. J. B 70, 343 (2009).

${ }^{28}$ See, e.g., J. A. Rodriguez, A. Etxeberria, L. González, and A. Maiti, J. Chem. Phys. 117, 2699 (2002). 